\title{
Treatment Outcome of Cisplatin-based Concurrent Chemoradio- therapy in the Patients with Locally Advanced Nasopharyngeal Cancer
}

Tae Hee Kim, M.D. ${ }^{1,7}$, Yoon Ho Ko, M.D. ${ }^{7}$, Myung Ah Lee, M.D., Ph.D. ${ }^{7}$, Bum-soo Kim, M.D., Ph.D. ${ }^{1,2}$, So Ryoung Chung, M.D., Ph.D. ${ }^{1,2}$, le Ryung Yoo, M.D. ${ }^{1,3}$, Chan-Kwon Jung, M.D., Ph.D. ${ }^{1,4}$, Yeon-Sil Kim, M.D., Ph.D. ${ }^{1,5}$, Min Sik Kim, M.D., Ph.D., ${ }^{1,6}$, Dong-II Sun, M.D., Ph.D., ${ }^{1,6}$, Young Seon Hong, M.D., Ph.D. ${ }^{7}$, Kyung Shik Lee, M.D., Ph.D. ${ }^{7}$ and Jin-Hyoung Kang, M.D., Ph.D. ${ }^{1,7}$

${ }^{1}$ Head \& Neck Cancer Interdisciplinary Team, Departments of ${ }^{2}$ Diagnostic Radiology, ${ }^{3}$ Nuclear Medicine, ${ }^{4}$ Hospital Pathology, ${ }^{5}$ Radiation Oncology, ${ }^{6}$ Otorhinolaryngology, ${ }^{7}$ Medical Oncology, The Catholic University of Korea College of Medicine, Seoul, Korea

Purpose: The standard treatment of locally advanced nasopharyngeal cancer is a concurrent chemoradiotherapy (CCRT), and cisplatin has been used as the most popular chemotherapeutic agent. But many different doses and schedules for cisplatin administration such as daily, weekly and 3 week cycles have been proposed. We compared and analyzed the tumor response, the overall survival, the toxicity and the chemotherapy dose intensity in the patients with locally advanced nasopharyngeal cancer who were treated with CCRT.

Materials and Methods: We performed a retrospective study on 55 patients with locally advanced nasopharyngeal cancer, and they were treated with CCRT as a front-line treatment from Jan 1996 to Jun 2007 at Kangnam Saint Mary's Hospital.

Results: The patients had a median age of 53 years (range: $19 \sim 75$ years). Of the total 55 patients, a 3-week cycle of $100 \mathrm{mg}$ cisplatin was administered in 31 patients and $30 \mathrm{mg}$ weekly cisplatin was administered in 24 patients combined with radiotherapy. Twenty one patients had a complete response and four patients had a partial response for a response rate of $71.4 \%(95 \% \mathrm{Cl}$ : $59.5 \sim 83.3$ ) after CCRT and followed by adjuvant chemo-

\section{INTRODUCTION}

Nasopharyngeal cancer is a kind of head and neck cancer, nevertheless, it is different from other head and neck cancer in the aspect of etiology, pathophysiology, response to treat-

Correspondence: Jin-Hyoung Kang, Division of Oncology, Department of Internal Medicine, Kangnam St. Mary's Hospital, The Catholic University of Korea, 505, Banpo-Dong, Seocho-Gu, Seoul 137-701, Korea. (Tel) 82-2-590-2711, (Fax) 82-2-533-8450, (E-mail) jinkang@ catholic.ac.kr

Received April 18, 2008, Accepted June 17, 2008 therapy. The complete response rates for the $\mathbf{3 0} \mathrm{mg}$ and $100 \mathrm{mg}$ cisplatin groups were $72.7 \%(95 \% \mathrm{Cl}: 54.9$ $90.5)$ and $54.2 \%(95 \% \mathrm{Cl}: 36.7 \sim 71.7)$, respectively ( $p=$ 0.23). The duration of CCRT in the $100 \mathrm{mg}$ cisplatin group was significantly longer than that of the $30 \mathrm{mg}$ cisplatin group (11.1 \pm 2.9 weeks vs. $9.0 \pm 1.2$ weeks, $p=0.003)$. The major deviation group, which was defined as prolongation of the radiotherapy duration for more than 2 weeks, had a significantly lower objective response rate than did the non-deviation group $(56.3 \%$ vs $84.2 \%$, respectively, $p=0.002)$. The major severe toxicities were leucopenia $(49.1 \%)$, pharyngoesophagitis $(49.1 \%)$, anorexia $(43.6 \%)$, nausea $(41.8 \%)$ and vomiting $(40 \%)$.

Conclusions: Weekly $30 \mathrm{mg}$ cisplatin-based CCRT is a practical, feasible cisplatin schedule for the patients with locally advanced nasopharyngeal cancer in regard to decreasing the interruption of radiation treatment and decreasing the treatment-related acute toxicities.

(Cancer Res Treat. 2008;40:62-70)

Key Words: Nasopharyngeal cancer, Chemoradiotherapy, Cisplatin, Objective response rate, Acute toxicities

ments, and prognosis. From the histological aspect, most head and neck cancers consist of more than $75 \%$ of highly differentiated squamous cell carcinoma. On the other hand, in nasopharyngeal cancer, the type 2 and 3 non-keratonizing epithelial carcinoma according to WHO classification, consists of more than $75 \%$. And Epstein-Barr virus (EBV) has been known to be deeply involved in the development of such histological nasopharyngeal cancer for a long time (1). Recently, it has been reported that the intake of food containing volatile nitrosamine and ethnic difference including the polymorphism of CYP2A6 gene involved in their metabolism plays an important role in the enhancement of the susceptibility to the development of nasopharyngeal cancer (2). Nasopharyngeal cancer is asymptomatic in early stage and thus it is mostly 
diagnosed in locally advanced stage. Compared with other head and neck cancer, local recurrence or distant metastasis occurs frequently even though aggressive treatments were performed. So 5-year survival rate has been known to be approximately $40 \sim 70 \%(1,3,4)$. However, the sensitivity to chemotherapeutic agents is relatively higher than other head and neck cancer, therefore, the concurrent chemoradiotherapy developed since the 1990s became an main therapeutic strategy to reduce local recurrence and distant metastasis as well as to improve overall survival (5).

Cisplatin is a representative cytotoxic chemotherapeutic agent, and it has been known that it acts as a radiation sensitizer and improves the antitumor efficacy of radiation therapy when combined with radiotherapy (6). Chemotherapuetic agents that have been attempted to combine with radiation therapy are methotrexate, hydroxyurea, 5-FU, bleomycin, etc. But they are not used any longer because they frequently induced severe toxicity such as mucositis, stomatitis, etc. And until now, only Cisplatin has been accepted as an agent that improves the efficacy of radiation therapy minimizing such an adverse events (7).

In the Intergroup 0099 study, Al-Sarraf et al proved the superiority of concurrent chemoradiotherapy (CCRT) in the aspect of progression-free survival and overall survival to radiation therapy alone (5). Afterward, even in Asian countries where nasopharyngeal cancer is prevalent, the treatment efficacy of concurrent chemoradiotherapy was confirmed in many clinical studies $(8,9,17)$. As a result of these studies, concurrent chemoradiotherapy followed by adjuvant combination chemotherapy with Cisplatin/5-fluorouracil has been accepted as a standard treatment for locally advanced nasopharyngeal cancer (10).

In most studies, the 5-year survival rate of concurrent chemoradiotherapy has been reported to be higher than $60 \%$, nevertheless, the fact that the consequent serious toxicity and the deterioration of the compliance to therapy lead to the failure of therapy ultimately has been underestimated $(11,12)$. In addition, in several clinical studies conducted after the Intergroup 0099 study, the administration schedule of Cisplatin was diverse and chemotherapeutic agents other than Cisplatin was administered, which could be considered indirect attempts to overcome the toxicity of concurrent chemoradiotherapy (13), nevertheless, studies investigating whether the toxicity could be reduced by the dose adjustment and schedule modification of Cisplatin are extremely limited. It is considered that in clinical practice, the frequency of interruption or withdrawal of treatment due to the severe toxicity of concurrent Cisplatinradiotherapy that has been used most widely in patients with nasopharyngeal cancer may be higher than reported data $(12,14)$ And we paid attentions on the fact that studies on the influence of such problems on the treatment outcome of concurrent chemoradiotherapy have not been performed sufficiently.

We investigated retrospectively the treatment outcome of two different cisplatin-based chemoradiotherapy in patients with locally advanced nasopharyngeal cancer. We evaluated the efficacy of treatment (tumor response rate, progression-free survival, and overall survival), and analyzed the effect of schedule modification of cisplatin on toxicity, compliance, and treatment outcomes.

\section{MATERIALS AND METHODS}

\section{1) Patients}

From January 1,1996 to June 30 , 2007, fifty five patients with locally advanced nasopharyngeal cancer were treated with concurrent chemoradiotherapy as a front-line treatment at Kangnam Saint Mary's hospital.

Eligible patients required histological diagnosis of keratonizing or non-keratonizing carcinoma (including differentiated or undifferentiated), tumor and nodal staging determined to be stage IIB, III, IVA, or IVB by American Joint Committee on Cancer (AJCC) 2002 classification. The lesions could be measured, patients had no evidence of systemic metastasis, were not diagnosed as other cancer in the past, and untreated with radiation or chemotherapy in the past. Computed tomography or magnetic resonance imaging on the nasopharynx and the cranial base area was performed in all patients for accurate response evaluation. If it is suspicious to have metastasis in the lung, bone, or liver, computed tomography, bone scan and other imaging test were performed to confirm them as well as biopsy if needed. In addition, to assess primary cancer as well as for biopsy, nasopharyngoscopy was performed at the time of diagnosis and the follow-ups. Prior to treatments, all patients received dental examination, and after the completion of appropriate treatments including tooth extract if required, they could receive CCRT.

\section{2) Radiation therapy}

The external radiotherapy technique was 2-dimensional conventional irradiation methods using a $4 \sim 15 \mathrm{MV}$ photon beam. The primary tumor site and adjacent tissues were treated to 70 Gy in 35 40 factions per $7 \sim 8$ weeks by a shrinking-field technique (Two lateral faciocervical fields to 50Gy in $25 \sim 28$ fractions per $5 \sim 5.5$ weeks followed by a three-field treatment fo 20 Gy in $10 \sim 11$ fractions per $2 \sim 2.2$ weeks). The single radiation dose was $1.8 \sim 2 \mathrm{~Gy}, 5$ times per week from Monday to Friday.

\section{3) Chemotherapy}

During radiotherapy, patients received cisplatin as planned. For $100 \mathrm{mg}$ Cisplatin group, cisplatin was administered three times every 3 weeks on days 1,22 , and 43 and for the $30 \mathrm{mg}$ cisplatin group, it was administered weekly on days $1,8,15$, $22,29,36,43$, and $50.0 .45 \%$ half normal saline containing $40 \mathrm{mEq}$ potassium chloride and $2,000 \mathrm{ml} 5 \%$ dextrose fluid were injected intravenously over 24 hours before and 24 hours after administration of Cisplatin and the second mannitol infusion. Cisplatin was injected intravenously in 60 minutes, and antiemetics such as metoclopramide, dexamethasone, and 5-HT antagonist were administered at 30 minutes before the administration of Cisplatin and then at 8 hours interval.

$100 \mathrm{mg}$ cisplatin was administered in most patients treated from 1996 to 2003 and $30 \mathrm{mg}$ cisplatin was administered in most patients treated since 2004. According to the result of response evaluation to concurrent chemoradiotherapy, subsequent chemotherapy was given for patients with objective response within 10 weeks after radiotherapy. $80 \mathrm{mg} / \mathrm{m}^{2}$ cisplatin was administered on day 1 and $1,000 \mathrm{mg} / \mathrm{m}^{2} 5$-FU was admini- 
stered on days 1 to 4 over 96 hours as an adjuvant chemotherapeutic agents. They were administered four times at 4 weeks interval and depending on the result of response evaluation performed 4 weeks after the completion of therapy, the followup observation or salvage chemotherapy was determined.

\section{4) Dose modification of chemotherapeutic agents}

If the absolute neutrolphil count was $\geq 1.5 \times 10^{9} / \mathrm{L}$ and the platelet count was $\geq 75 \times 10^{9} / \mathrm{L}$, no dose modification was made. If the absolute neutrophil count was $1,000 \sim 1,499 / \mu \mathrm{L}$ and/or the platelet count was $50,000 \sim 74,999 / \mu \mathrm{L}$, dose of cisplatin was reduced to $80 \%$. If the absolute neutrophil count was less than $1,000 / \mu \mathrm{L}$ and/or the platelet count was less than $50,000 / \mu \mathrm{L}$, chemotherapy was withheld until the WBC count and the platelet count were greater than $3,000 / \mu 1$ and $100,000 / \mu \mathrm{L}$, respectively. The dose of cisplatin was adjusted according to the value of serum creatinine measured just before chemotherapy infusion at day 1 . If the creatinine concentration was $<1.5 \mathrm{mg} / \mathrm{dl}$, no dose adjustment was required. If the creatinine concentration increased to $1.5 \sim 3.0 \mathrm{mg} / \mathrm{dl}$, the dose of cisplatin was reduced to $80 \%$, or the administration of Cisplatin was withheld, 24 hours urine chemistry test was performed, and administration of chemotherapy was determined by creatinine clearance (if higher than $45 \mathrm{ml} / \mathrm{min}$, dose of cisplatin reduced to $80 \%$, and if lower than $45 \mathrm{ml} / \mathrm{min}$, the administration was withheld.). For cases whose creatinine was elevated to higher than $3.0 \mathrm{mg} / \mathrm{dl}$ continuously, cisplatin was no longer administered, and radiotherapy alone was continued.

\section{5) Evaluation of tumor response}

The primary end point of our study was response rate. For the evaluation of the response to concurrent chemoradiotherapy, tumor response criteria of the World Health Organization (WHO) was applied. Complete response (CR) was defined as disappearance of all objective evidence of disease confirmed by physical examination, direct nasopharyngoscopy and imaging, partial response (PR) was defined as a decrease of the sum of the products of longest and its perpendicular diameter in all measurable lesions by at least $50 \%$, progressive disease (PD) was defined as the appearance of new lesion or an increase of the sum of the products of the longest and its perpendicular diameter in all measurable lesions by more than $25 \%$, and stable disease (SD) was all cases who did not belong to the above mentioned 3 categories. For the evaluation of tumor response, physical examination and nasopharyngoscopy were performed, and for objective evaluation, computed tomography (CT) or magnetic resonance imaging (MRI) were performed. If recurrence was suspected, the suspicious lesion was confirmed by PET-CT, and biopsy was performed if needed. The first response evaluation was performed 4 weeks after the completion of concurrent chemoradiotherapy, and the second response evaluation was performed just before adjuvant chemotherapy, and the third final response evaluation was performed after the completion of 3 to 4 times of adjuvant chemotherapy. Disease was evaluated every 2 months during the first year, every 3 months for second and third year, and every 6 months afterward. Rehabilitation evaluation on articulation or swallowing as well as treatment was performed if required. The secondary end point was progression-free survival and overall sur- vival. The progression-free survival was defined as the time from the initiation of treatment to the date of first observation of progressive disease or death due to any cause. Overall survival was defined as the date from initiation of treatment to death due to any cause.

\section{6) Toxicity}

The tertiary end points of our study were toxicity grading and dose intensity of the radiation and cisplatin. Toxicity was evaluated during concurrent chemoradiotherapy and adjuvant chemotherapy as well as 1 month after the completion of all treatment and dose intensity was evaluated by calculating the days when the treatment was interrupted due to toxicity. Acute toxicity was evaluated based on the National Cancer Institutecommon toxicity criteria, ver. 2.0 (NCI-CTC) and the acute morbidity scoring criteria of Radiation Therapy Oncology Group (RTOG) (16).

\section{7) Statistical analysis}

Progression-free survival and overall survival were estimated by Kaplan-Meier method, and all $\mathrm{p}$ values were based on two-sided test. Cox regression models were used to assess for important prognostic factors including performance status (Eastern Cooperative Oncology Group (ECOG) scale), primary tumor stage, lymph node stage, WHO histological classification, blood test values prior to treatment (hemoglobin, LDH, albumin), the frequency of the administration of granulocytecolony stimulating factor (G-CSF), the frequency of transfusion and the frequency of febrile neutropenia. And the impact of these factors on tumor response rate, progression-free survival and overall survival was validated using multivariate Cox regression model and log-rank test. Response and toxicity among subgroups were compared and analyzed using chisquare test and Fisher's exact test. Statistical analysis was performed using the SAS statistic package (version 8.2), and $p$ value less than 0.05 was statistically significant.

\section{RESULTS}

\section{1) Clinical characteristics of patients}

Among fifty five patients treated with concurrent chemoradiotherapy, fifty four patients were evaluable. Response evaluation could not be performed in one patient who was dropped out during the treatment due to serious mucositis and decreased performance status. Thirty nine Patients were treated with adjuvant chemotherapy after concurrent chemoradiotherapy. Among fifteen patients who did not receive adjuvant chemotherapy, thirteen patients either refused the therapy or dropped out during the follow-up observation, one patient could not receive the further treatment due to the grade 3 renal toxicity developed after concurrent chemoradiotherapy, and the remaining one patient underwent cyberknife surgery on the primary lesion.

Thirty five patients could complete the planned adjuvant chemotherapy after CCRT and the final response evaluation could be performed. Among four patients who could not be evaluated, one patient was lost in the follow-up and the rest three patients were under adjuvant chemotherapy currently, and 
their evaluation was not performed yet.

Among total fifty five patients, thirty one patients received with $100 \mathrm{mg}$ cisplatin at 3 weeks interval during CCRT, and 24 patients received $30 \mathrm{mg}$ cisplatin weekly. The stage of primary tumor, the stage of lymph nodes, WHO histological classification, performance status and other clinical factors associated with progonosis were not significantly different between the two groups except age, that is, patient's age of 30 $\mathrm{mg}$ Cisplatin group was significantly higher than that of 100 mg Cisplatin group $(\mathrm{p}=0.016)$ (Table 1).

\section{2) Tumor response}

The WHO response criteria were followed strictly. The result of the response evaluation performed after CCRT was as

Table 1. Patient characteristics

\begin{tabular}{|c|c|c|c|c|c|}
\hline \multirow{2}{*}{$\begin{array}{c}\text { Characteristics } \\
\text { And clinical } \\
\text { variables }\end{array}$} & \multicolumn{2}{|c|}{$\begin{array}{l}3 \text { weeks interval cisplatin } \\
100 \mathrm{mg} / \mathrm{m}^{2} \quad(\mathrm{n}=31)\end{array}$} & \multicolumn{2}{|c|}{$\begin{array}{l}\text { Weekly cisplatin } \\
30 \mathrm{mg} / \mathrm{m}^{2} \quad(\mathrm{n}=24)\end{array}$} & \multirow{2}{*}{$\mathrm{p}$ value } \\
\hline & No. & $\%$ & No. & $\%$ & \\
\hline Age (years) & \multicolumn{2}{|c|}{$49(19 \sim 68)$} & \multicolumn{2}{|c|}{$56.5(32 \sim 74)$} & 0.016 \\
\hline \multicolumn{6}{|l|}{ Gender } \\
\hline $\mathrm{M}: \mathrm{F}$ & $24: 7$ & $77.4: 22.6$ & $16: 8$ & $66.7: 33.3$ & $\mathrm{NS}^{\dagger}$ \\
\hline ECOG PS & & & & & $\mathrm{NS}^{\dagger}$ \\
\hline 0 & 12 & 38.7 & 8 & 33.3 & \\
\hline 1 & 19 & 61.3 & 14 & 58.3 & \\
\hline 2 & 0 & 0 & 2 & 8.3 & \\
\hline \multicolumn{5}{|l|}{ T stage* } & $\mathrm{NS}^{\dagger}$ \\
\hline $\mathrm{T} 1$ & 5 & 9.7 & 4 & 16.7 & \\
\hline $\mathrm{T} 2$ & 8 & 25.8 & 6 & 25.0 & \\
\hline $\mathrm{T} 3$ & 7 & 22.6 & 2 & 8.3 & \\
\hline $\mathrm{T} 4$ & 11 & 35.5 & 12 & 50.0 & \\
\hline \multicolumn{5}{|l|}{$\mathrm{N}$ stage* } & $\mathrm{NS}^{\dagger}$ \\
\hline No & 5 & 9.7 & 3 & 12.5 & \\
\hline $\mathrm{N} 1$ & 10 & 32.3 & 7 & 29.2 & \\
\hline $\mathrm{N} 2$ & 13 & 41.9 & 11 & 45.8 & \\
\hline N3 & 3 & 9.7 & 3 & 12.5 & \\
\hline \multicolumn{5}{|l|}{ Stage* } & $\mathrm{NS}^{\dagger}$ \\
\hline IIb & 5 & 16.7 & 4 & 16.7 & \\
\hline III & 13 & 41.9 & 5 & 20.8 & \\
\hline IV & 13 & 41.9 & 15 & 62.5 & \\
\hline \multicolumn{5}{|l|}{ Histology } & $\mathrm{NS}^{\dagger}$ \\
\hline WHO Gr2/3 & 24 & 77.4 & 18 & 75 & \\
\hline Hemoglobin (g/dl) & \multicolumn{2}{|c|}{$13.4 \pm 1.4$} & \multicolumn{2}{|c|}{$13.1 \pm 1.6$} & $\mathrm{NS}_{+}^{\dagger}$ \\
\hline Albumin (g/dl) & \multicolumn{2}{|c|}{$4.0 \pm 0.3$} & \multicolumn{2}{|c|}{$4.1 \pm 0.4$} & $\mathrm{NS}^{\dagger}$ \\
\hline $\mathrm{LDH}(\mathrm{U} / \mathrm{L})$ & \multicolumn{2}{|c|}{$314.2 \pm 82.3$} & \multicolumn{2}{|c|}{$363.5 \pm 114.0$} & $\mathrm{NS}^{\dagger}$ \\
\hline
\end{tabular}

*AJCC 2002 staging system, ${ }^{\dagger}$ non-significant.

Table 2. Antitumor efficacy; Response after concurrent chemoradiotherapy (CCRT) and adjuvant chemotherapy (AC)

\begin{tabular}{|c|c|c|c|c|c|c|c|c|}
\hline & & \multicolumn{2}{|c|}{ Cispltin $100 \mathrm{mg}$} & \multicolumn{2}{|c|}{ Cisplatin $30 \mathrm{mg}$} & \multicolumn{2}{|c|}{ Total patients } & \multirow{2}{*}{$\mathrm{p}$ value } \\
\hline & & No. & $\%$ & No. & $\%$ & No. & $\%$ & \\
\hline \multirow[t]{2}{*}{$\mathrm{CR}^{*}$} & CCRT & 11 & 35.5 & 12 & 52.2 & 23 & 42.6 & 0.20 \\
\hline & $\mathrm{AC}$ & 13 & 54.2 & 8 & 72.7 & 21 & 60.0 & 0.23 \\
\hline \multirow[t]{2}{*}{$\mathrm{PR}^{\dagger}$} & CCRT & 20 & 64.5 & 10 & 43.5 & 30 & 52.6 & \\
\hline & $\mathrm{AC}$ & 3 & 12.5 & 1 & 9.1 & 4 & 11.4 & \\
\hline \multirow[t]{2}{*}{$\mathrm{SD}^{\ddagger}$} & CCRT & 0 & 0 & 1 & 4.3 & 1 & 1.9 & \\
\hline & $\mathrm{AC}$ & 0 & 0 & 1 & 9.1 & 1 & 2.8 & \\
\hline \multirow[t]{2}{*}{$\mathrm{PD}^{\S}$} & CCRT & 0 & 0 & 0 & 0 & 0 & 0 & \\
\hline & $\mathrm{AC}$ & 8 & 33.3 & 1 & 9.1 & 9 & 25.7 & \\
\hline
\end{tabular}

${ }^{*}$ complete response, ${ }^{\dagger}$ partial response, ${ }^{\dagger}$ stable disease, ${ }^{\S}$ progressive disease. 
followed; CR 23 patients $(42.6 \%, 95 \%$ CI $29.5 \sim 55.7)$, PR 30 patients $(55.6 \%, 95 \%$ CI $42.5 \sim 68.7)$, and SD 1 patient $(1.9 \%$ 95\%, CI 0 5.5) (Table 2). Compared $100 \mathrm{mg}$ cisplatin group with $30 \mathrm{mg}$ cisplatin group, complete response was 11 patients $(35.5 \%, 95 \%$ CI $18.7 \sim 52.3)$ and 12 patients $(52.2 \%, 95 \%$ CI $32.2 \sim 72.2$ ), respectively and a higher complete rate was observed in the $30 \mathrm{mg}$ group without statistically significance $(p=0.20)$. Subsequent adjuvant chemotherapy following CCRT, 5-FU/Cisplatin combination therapy was performed in most cases $(88 \%)$. All patients of the $30 \mathrm{mg}$ cisplatin group were treated with 5-FU and Cisplatin, the other hand, among 24 patients of the $100 \mathrm{mg}$ cisplatin group, 4 patients were treated with chemotherapeutic agents other than 5-FU and Cisplatin (5-FU+carboplatin in one patient, 5-FU+Cisplatin+Bleomycin in two patients, 5-FU+ Cisplatin+Docetaxel in one patient). The result of the final response evaluation after the completion of adjuvant chemotherapy was as followed; CR 21 patients (60.0\%, 95\% CI 44 76), PR 4 patients $(11.4 \%$, 95\% CI $0.9 \sim$ 21.9), and objective response rate $71.4 \%$ (95\% CI 56.4 86.4).

Compared the $100 \mathrm{mg}$ cisplatin group with the $30 \mathrm{mg}$ group, complete response was 13 patients $(54.2 \%, 95 \%$ CI $34.2 \sim 74.2)$ and 8 patients $(72.7 \%, 95 \%$ CI $46.4 \sim 99)$, respectively, and it was shown to be $18.5 \%$ higher in the $30 \mathrm{mg}$ administration group without statistical significance $(\mathrm{p}=0.23)$ (Table 2$)$. On the other hand, performance status, the values of blood test performed prior to treatments (hemoglobin, albumin, LDH), the frequency of the administration of granulocyte colony-stimulatory factor, the number of transfusion, the frequency of febrile neutropenia, etc. between the two groups were not significantly different, and statistical analyses showed that they did not influences on tumor response.

\section{3) Radiation therapy}

The mean radiation dosage of the entire patients received concurrent chemoradiotherapy was $68.4 \pm 6.0$ Gy $(43.2 \sim 75.6$ $\mathrm{Gy}$, the $100 \mathrm{mg}$ cisplatin group $68.2 \pm 5.5 \mathrm{~Gy}$, the $30 \mathrm{mg}$ cisplatin group $68.4 \pm 6.0 \mathrm{~Gy}$ ), and the mean treatment duration was $10 \pm 2.5$ weeks $(7.3 \sim 19.7)$. However, the mean treatment duration of the $100 \mathrm{mg}$ cisplatin group was $11.0 \pm 2.9$ weeks (7.4 $\sim 19.7$ ), the $30 \mathrm{mg}$ group was $9.2 \pm 1.2$ weeks $(7.3 \sim 12.1)$, and the treatment duration was significantly prolonged in the 100 mg Cisplatin group $(p=0.003)$. In the entire patients, the

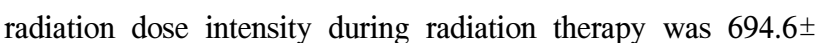
$126.5 \mathrm{cGy} /$ week. However, the radiation dose intensity of the $100 \mathrm{mg}$ cisplatin group and the $30 \mathrm{mg}$ group was shown to be $642.5 \pm 130.8 \mathrm{cGy} /$ week and $768.1 \pm 122.1 \mathrm{cGy} /$ week, respectively, therefore the $30 \mathrm{mg}$ cisplatin group showed a significantly higher radiation dose intensity $(\mathrm{p}=0.0008)$.

\section{4) Cisplatin chemotherapy}

In the comparison whether the planned chemotherapeutic agents were administered during the concurrent chemoradiotherapy from the aspect of the frequency of the administration of chemotherapeuytic agents, in the $100 \mathrm{mg}$ cisplatin group, patients who received all 3 times were 26 patients out of 31 patients $(84 \%)$, and the remaining 5 patients were less than 3 times. In the $30 \mathrm{mg}$ cisplatin group, patients received more than 7 times were 22 patients out of 24 patients (92\%), and only the remaining 2 patients were less than 7 times. Among the total patients received concurrent chemoradiotherapy, the mean value of the Cisplatin administration dose was $250 \pm 64.8 \mathrm{mg} / \mathrm{m}^{2}$ (60 $460 \mathrm{mg} / \mathrm{m}^{2}$ ), and the $100 \mathrm{mg}$ cisplatin group and the $30 \mathrm{mg}$ group was $273.5 \pm 64.4 \mathrm{mg} / \mathrm{m}^{2}$ and $218.8 \pm 51.9 \mathrm{mg} / \mathrm{m}^{2}$, respectively. On the other hand, the mean dose intensity of cisplatin during concurrent chemoradiotherapy was $25.1 \pm 6.4 \mathrm{mg} / \mathrm{m}^{2}$ / week. The dose intensity of the $100 \mathrm{mg}$ cisplatin group and the $30 \mathrm{mg}$ group was $25.7 \pm 6.7 \mathrm{mg} / \mathrm{m}^{2} /$ week and $24.3 \pm 6.2 \mathrm{mg} /$ $\mathrm{m}^{2} /$ week, respectively without statistical significance $(\mathrm{p}=0.44)$. In addition, during concurrent chemoradiotherapy, 15 patients required dose reduction of Cisplatin more than once in the 100 $\mathrm{mg}$ group, but none of patients in the $30 \mathrm{mg}$ group. As the causes of dose reduction of cisplatin, severe pharyngoesophagitis (15 patients), decreased performance status (4 patients), leucopenia (4 patients), severe nausea/vomiting (3 patients) and renal toxicity (1 patient) were noted (causes were overlapped).

\section{5) Toxicity profile}

Severe toxicity developed in one patient among the $100 \mathrm{mg}$ cisplatin group during concurrent chemoradiotherapy. The treatment of this patient was discontinued due to renal toxicity (grade 3) and anemia (grade 2). Although renal and bone marrow function were recovered by conservative treatment; aggressive chemotherapy could not be continued afterward.

In concurrent chemoradiotherapy, the grade 3 or 4 serious

Table 3. Acute toxicity during concurrent chemoradiotherapy

\begin{tabular}{|c|c|c|c|c|c|c|c|c|c|}
\hline \multirow{3}{*}{ Toxicity } & \multicolumn{9}{|c|}{ Toxicity grade } \\
\hline & \multicolumn{4}{|c|}{ Cisplatin $100 \mathrm{mg}(\mathrm{n}=31)$} & \multicolumn{4}{|c|}{ Cisplatin $30 \mathrm{mg}(\mathrm{n}=24)$} & \multirow{2}{*}{$\mathrm{p}$ value } \\
\hline & 0 & 3 & 4 & Severe $(\%)$ & 0 & 3 & 4 & Severe $(\%)$ & \\
\hline leukopenia & 2 & 17 & 1 & $18(58.1)$ & 4 & 9 & 0 & $9(37.5)$ & 0.29 \\
\hline Anemia & 22 & 2 & 1 & $3(8.8)$ & 19 & 0 & 0 & $0(0)$ & 0.25 \\
\hline Thrombocytopenia & 23 & 3 & 1 & $4(12.9)$ & 17 & 2 & 0 & $2(8.3)$ & 0.69 \\
\hline Skin rash & 24 & 1 & 0 & $1(3.2)$ & 13 & 0 & 0 & $0(0)$ & 0.99 \\
\hline Nausea & 4 & 15 & 0 & $15(48.1)$ & 1 & 8 & 0 & $8(33.3)$ & 0.78 \\
\hline Vomiting & 7 & 12 & 2 & $14(45.2)$ & 8 & 8 & 0 & $8(33.3)$ & 0.46 \\
\hline Pharyngoesophagitis & 4 & 12 & 3 & $15(48.1)$ & 2 & 12 & 0 & $12(50)$ & 0.75 \\
\hline Anorexia & 2 & 14 & 0 & $14(48.1)$ & 2 & 10 & 0 & $10(41.7)$ & 0.82 \\
\hline Neuropathy & 21 & 0 & 0 & $0(0)$ & 14 & 0 & 0 & $0(0)$ & 1.0 \\
\hline
\end{tabular}


toxicity was as followed; leucopenia and pharyngoesophagitis (27 patients, $49.1 \%$ ) each followed by anorexia (24 patients, $43.6 \%$ ), nausea (23 patients, $41.8 \%$ ), vomiting (22 patients, $40 \%$ ), thrombocytopenia (6 patients, $10 \%$ ), anemia (3 patients, $5.5 \%$ ), and dermatitis (1 patient, $1.8 \%$ )(Table 3). All serious toxicities were observed more frequently in the $100 \mathrm{mg}$ cisplatin group than the $30 \mathrm{mg}$ cisplatin group except pharyngoesophagitis, but there is no statistically significant difference between the two groups (Table 3).

Afterward, in the subsequent adjuvant chemotherapy, as the grade 3 or 4 serious toxicity, leucopenia (17 patients, 57\%), anemia (3 patients, 11.4\%), thrombocytopenia (8 patients, $31.4 \%$ ), nausea (4 patients, $11.4 \%$ ), vomiting (3 patients, $8.6 \%$ ), anorexia (7 patients, $20 \%$ ), mucositis (6 patients, $17.1 \%)$, and neurotoxicity (2 patients, $5.7 \%$ ) were noted. All toxicities were more frequent in the $100 \mathrm{mg}$ cisplatin group except neurotoxicity, and particularly, serious nausea, vomiting and mucositis were observed only in the $100 \mathrm{mg}$ cisplatin group. However, adjuvant chemotherapeutic agents were somewhat different between the two groups, and thus it is difficult to conclude that there were significant differences in toxicity profile between two groups. All patients of $30 \mathrm{mg}$ cisplatin group were treated with 5-FU and cisplatin, however, among 24 patients of the 100 mg group, 4 patients were treated with chemotherapeutic agents other than 5-FU and cisplatin (5-FU+carboplatin in one patient, 5-FU+Cisplatin+Bleomycin in two patient, 5-FU+Cisplatin+ Docetaxel in one patient), and it was difficult to consider that accurate toxicity evaluation was done due to the limitation of retrospective analysis (Table 4).

\section{6) Tumor response on the prolongation of treatment duration}

Due to the serious toxicity developed during concurrent chemoradiotherapy, the planned radiation therapy was interrupted or prolonged. In particular, the major deviation prolonged more than 2 weeks was observed in 24 patients (44.4\%) of entire 55 patients, and among them, 20 patients (83.3\%) were observed in the $100 \mathrm{mg}$ cisplatin group and 4 patients $(16.7 \%)$ were observed in the $30 \mathrm{mg}$ group $(\mathrm{p}=0.0006)$ (Fig. 1). Among 24 patients who showed the major deviation, in 16 patients whose final response rate could be evaluated, complete response and partial response were 6 patients $(37.5 \%, 95 \% \mathrm{CI}$ $13.8 \sim 61.2)$ and 3 patients $(18.8 \%, 95 \%$ CI $0 \sim 37.9)$, respectively, and the objective response rate was $56.3 \%$ (95\% CI 32 80.6). On the other hand, among 19 patients whose treatment was completed within the planned treatment period or the

Table 4. Acute toxicity during adjuvant chemotherapy

\begin{tabular}{|c|c|c|c|c|c|c|c|c|}
\hline \multirow{3}{*}{ Toxicity } & \multicolumn{8}{|c|}{ Toxicity grade } \\
\hline & \multicolumn{4}{|c|}{ Cisplatin $100 \mathrm{mg}(\mathrm{n}=24)$} & \multicolumn{4}{|c|}{ Cisplatin $30 \mathrm{mg} \quad(\mathrm{n}=11)$} \\
\hline & 0 & 3 & 4 & Severe $(\%)$ & 0 & 3 & 4 & Severe $(\%)$ \\
\hline leucopenia & 2 & 11 & 4 & $15(62.5)$ & 0 & 3 & 2 & $5(45.5)$ \\
\hline Anemia & 8 & 3 & 0 & $3(12.5)$ & 4 & 1 & 0 & $1(9.1)$ \\
\hline Thrombocytopenia & 12 & 7 & 1 & $8(33.3)$ & 5 & 3 & 0 & $3(27.3)$ \\
\hline Dermatitis & 24 & 0 & 0 & $0(0)$ & 11 & 0 & 0 & $0(0)$ \\
\hline Nausea & 0 & 3 & 1 & $4(16.7)$ & 3 & 0 & 0 & $0(0)$ \\
\hline Vomiting & 11 & 2 & 1 & $3(12.5)$ & 9 & 0 & 0 & $0(0)$ \\
\hline Mucositis & 4 & 5 & 1 & $6(25)$ & 0 & 0 & 0 & $0(0)$ \\
\hline Anorexia & 1 & 5 & 0 & $5(20.8)$ & 0 & 2 & 0 & 2 (18.2) \\
\hline Neuropathy & 9 & 1 & 0 & $1(4.2)$ & 2 & 1 & 0 & $1(9.1)$ \\
\hline
\end{tabular}

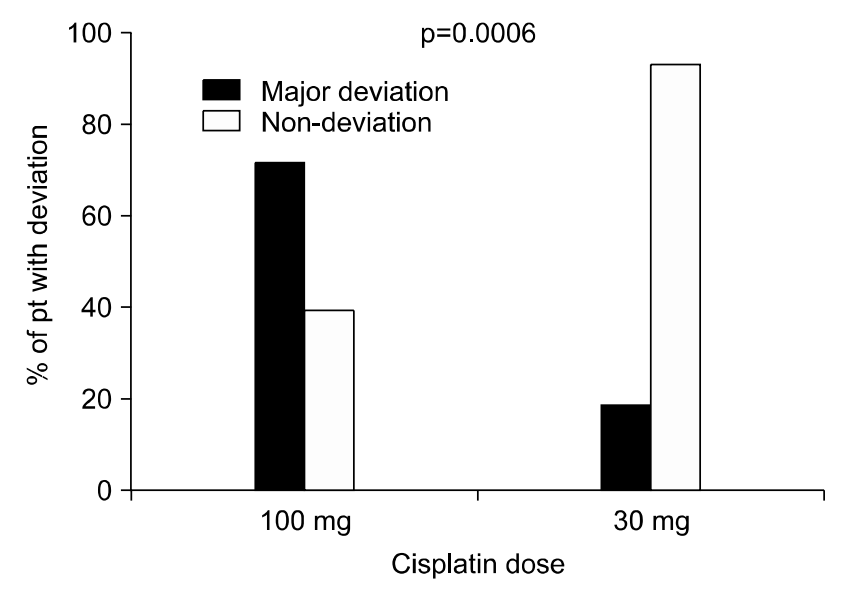

Fig. 1. Major radiation deviation on cisplatin schedule.

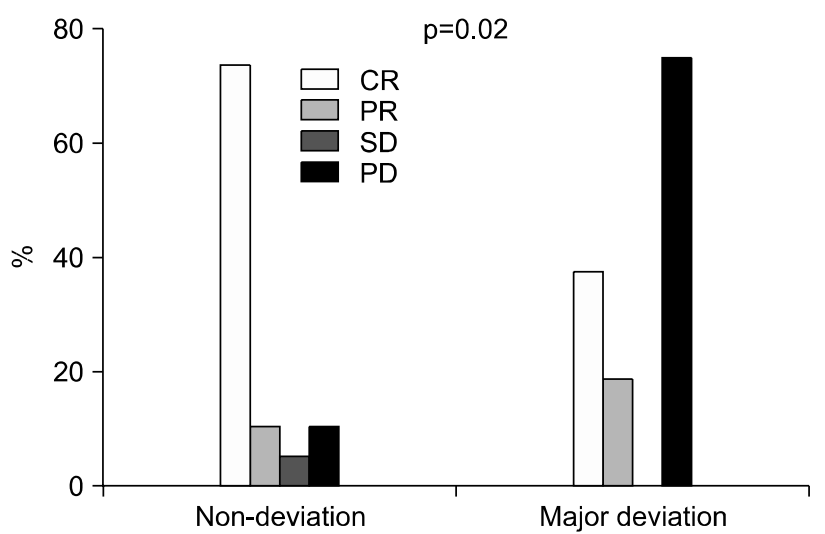

Fig. 2. Response difference between major deviation group (MD) and non-deviation group (ND). 
prolonged period was less than 2 weeks, complete response and partial response was 14 patients and $(73.7 \%, 95 \%$ CI $53.9 \sim$ 93.5) and 2 patients $(10.5 \%, 95 \%$ CI $0 \sim 24.3)$, respectively, and the objective response rate was $84.2 \%$ (95\% CI $67.8 \sim$ $100)(p=0.02)$ (Fig. 2). Among the patients whose disease progression was confirmed, 7 patients $(43.8 \%)$ in major deviation group and 2 patients $(10.5 \%)$ in non-deviation group were documented with marked difference between the two groups.

\section{7) Progression-free survival and overall survival}

The median follow-up duration of the entire patients was 57 months $(3.0 \sim 149.5)$. Compared with 80 months $(4.2 \sim 104.7)$ of the $100 \mathrm{mg}$ cisplatin group, the $30 \mathrm{mg}$ cisplatingroup was 19.4 months (3.0 81.2), and the follow-up duration of the 30 $\mathrm{mg}$ cisplatin group was significantly shorter $(\mathrm{p}<0.001)$. The median overall survival of the entire 55 patients was 51.3 months $(3.0 \sim 149.5)$, 3-year survival rate was $60 \%$, and 5 -year survival rate was $47 \%$ (Fig. 3). The median progression-free survival was 30.1 months $(3.0 \sim 149.5)$, and 3-year progressionfree survival was $27 \%$.

Recurrence occurred in 16 patients (29.1\%). It was confirmed during follow-up period. Among them, local recurrence in 8 patients $(14.5 \%)$, distant metastasis in 7 patients $(12.7 \%)$ and

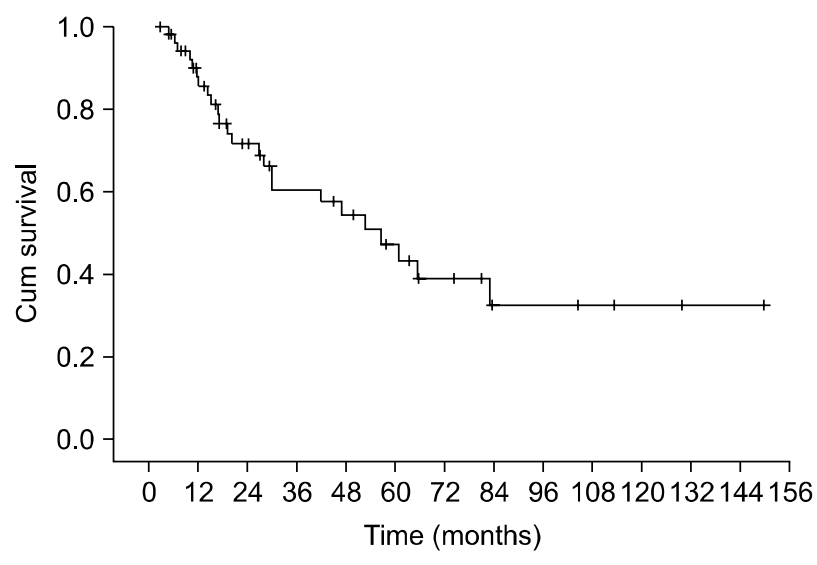

Fig. 3. Overall survival of nasopharyngeal cancer.

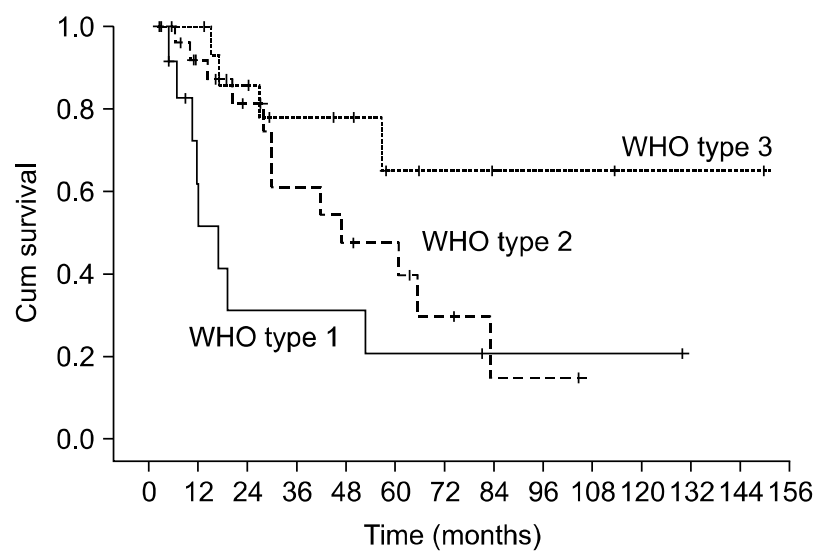

Fig. 4. overall survival time for 3 different WHO histology types. local recurrence and distant metastasis were detected simultaneously in 1 patient (1.8\%). Among 16 recurred patients, 14 patients were in the $100 \mathrm{mg}$ cisplatin group, but, their median follow-up duration was greatly longer than the $30 \mathrm{mg}$ cisplatin group, and thus it was difficult to conclude that more frequent recurrence occurred in the $100 \mathrm{mg}$ cisplatin group. In terms of the association of the prolongation of the radiation duration and recurrence, the interruption day of radiation therapy was $20.4 \pm$ 15.1 days in recurrence group and $14.6 \pm 11.8$ days in nonrecurrence group. It was postulated that the interruption of radiation therapy was correlated with disease recurrence with borderline significance $(\mathrm{p}=0.06)$. On the other hand, analyses showed that performance status, the values of blood test prior to treatment (hemoglobin, albumin, $\mathrm{LDH}$ ), the frequency of the administration of granulocyte colony-stimulatory factor, the frequency of febrile neutropenia, etc. did not affect overall survival (data not shown). However, in terms of WHO histology classification, 5-year survival rate was highest in WHO type 3 (undifferentiated, 65\%), and was lowest in WHO type 1 (keratinizing, 20\%) ( $\mathrm{p}=0.02$, Fig. 4) $(20,21)$.

\section{DISCUSSION}

The superiority of concurrent chemoradiotherapy that has been accepted as the standard treatment in patients with locally advanced nasopharyngeal cancer has been already proven by a meta-analysis reported in 2006 (10).

However, until now, the fundamental method to ameliorate serious acute toxicity induced by concurrent chemoradiotherapy has not been shown yet. At the present time when concurrent chemoradiotherapy has been accepted widely as a standard treatment, it is considerd that efforts to minimize serious acute toxicity are required by schedule modification of cisplatin or radiation technique.

In current study, tumor response rate, progression-free survival, overall survival and other treatment outcomes such as toxicity profile, major deviation due to acute toxicity on tumor response were analyzed retrospectively in patients with locally advanced nasopharyngeal cancer who were treated with concurrent chemoradiotherapy as a front-line treatment. In clinical study reported by Al-Saraff et al., the compliance of concurrent chemoradiotherapy was only $63 \%$ (5), and it has been revealed by several clinical studies that the compliance to concurrent chemoradiotherapy could not reach the level in most Asian patients, especially Southern China where nasopharyngeal cancer is prevalent. According to the study reported by Chitapanarux et al., when the dosage of chemotherapeutic agents as well as the schedule identical to the intergroup 0099 were applied to Thai patients, the completion rate of concurrent chemoradiotherapy was merely 59\%, and among patients completed concurrent chemoradiotherapy, the patients who completed 3 times adjuvant chemotherapy were only $26 \%$ of the entire patients (14). Such research results could not be explained as a ethnic difference limited to Asia where nasopharyngeal cancer occurs prevalently, because the completion rate of concurrent chemoradiotherapy was as low as $43 \%$ in clinical study performed in Canada (18). To overcome such problems, the administration schedule of cisplatin was changed from $100 \mathrm{mg} 3$ weeks to 30 
mg weekly regimen. As a result, showed that the severity and frequency of acute toxicity were reduced noticeably, and thus it was found that the patients's compliance was much improved when compared with $100 \mathrm{mg}$ at 3 weeks interval schedule. Although the improvement of compliance was associated with the frequency and dose of Cisplatin administered, it could be confirmed by the shortening of radiation interruption duration. In other words, the major deviation of radiation treatment was observed frequently in the $100 \mathrm{mg}$ cisplatin group, and it was implicated that such results influence on the deterioration of tumor response (Fig. 1 and 2). Toxicity profile was reduced slightly in the $30 \mathrm{mg}$ Cisplatin group, but it was not statistically significant. In addition, performance status, the values of blood test performed prior to treatments (hemoglobin, albumin, $\mathrm{LDH}$ ), the frequency of granulocyte colony-stimulatory factor, the frequency of transfusion, the frequency of febrile neutropenia, etc. between the two groups were not different, and analyses showed that they did not influence on overall survival (data not shown). The dose intensity of cisplatin of the two groups during the concurrent chemoradiotherapy was not significantly different, on the other hand, the fact that the dose intensity of radiation therapy was significantly higher in the 30 $\mathrm{mg}$ cisplatin group than the $100 \mathrm{mg}$ Cisplatin group, is thought to be the result of study validating that the reduction of radiation interruption played a critical role in the improvement of treatment outcomes is spite of higher mean age. Already, Kwong et al. have reported that in locally advanced nasopharyngeal cancer, prolongation of radiation therapy lead to poor prognosis, which was due to repopulation of tumor cells (11). However, in our study, significant reduction of interrupted days of radiation therapy by weekly schedule modification of cisplatin, tumor response rate could be improved. Nevertheless, the objective proof whether it improved progression-free survival or overall survival could not be obtained. Because the follow-up duration of the $100 \mathrm{mg}$ cisplatin group and the 30 $\mathrm{mg}$ cisplatin group were greatly different. So it was no sense to compare the two groups. It is thought that after enough time to follow-up, its accurate analysis could be performed in the near future.

On the other hand, the response rate after concurrent chemoradiotherapy shows a great diversity in different clinical studies. In other words, in the Intergroup 0099 study, the complete response rate was $49 \%$, and in other studies performed in Singapore and Taiwan, it was $83 \%$ and $95 \%$, respectively, and it is difficult to conclude that tumor response itself has a absolute effects on progression-free survival or overall survival $(5,8,9)$. In addition, in the evaluation of tumor response, the judgment of radiologist, radiation oncologist, and otorhinolaryngologists does not concordant in some cases. For the evaluation of response of head and neck cancer by imagings, it is thought that discriminative evaluation criteria that could be distinguished from other organs are required, and for the use of CT, MRI, PET-CT, and other diagnostic tools, appropriate principles are required.

Most acute toxicities except pharyngoesophagitis were developed frequently in the $100 \mathrm{mg}$ cisplatin group without statistical significance. Pharyngoesophagitis induces anorexia, nausea, and vomit, which leads to weight reduction and the deterioration of performance status, and thus it acts as a major factor causing the interruption or delay of treatments. To prevent this, education prior to radiation treatment, sufficient gargling and prophylactive tube feeding are of help (19). As the reasons that pharyngoesophagitis was shown to be somewhat higher in the $30 \mathrm{mg}$ cisplatin group, it is thought that firstly, the mean age of the $30 \mathrm{mg}$ cisplatin group was significantly higher than that of the $100 \mathrm{mg}$ group, secondly, in comparison with the $100 \mathrm{mg}$ cisplatin group, in the $30 \mathrm{mg}$ cisplatin group, the opportunity to evaluate toxicity was relatively more frequent due to weekly administration, thirdly, in the $30 \mathrm{mg}$ cisplatin group, not only interrupted day of radiation therapy was relatively shorter than the $100 \mathrm{mg}$ group, but also cisplatin admiministered weekly suppresses the recovery of cells damaged by radiation therapy continuously, which delays the time required to recover to normal cells (24).

On the other hand, the only prognostic factor on overall survival was the WHO histology classification, that is type 3 showed the best prognosis. In countries including China where nasopharyngeal cancer occurs prevalently, the WHO type 3 consists of more than $90 \%$ of the entire nasopharyngeal cancer, hence, by developing more aggressive treatment methods for them, high survival rates may be expected.

It is thought that our study showed intriguing results that the treatment outcome of concurrent chemoradiotherapy was improved by schedule modification of cisplatin. but, the evaluation of quality of life or toxicity was not performed sufficiently because of the limitation of retrospective studies, and the number of patients, age, and the follow-up duration etc. were also greatly different between the two different cisplatin groups. We attained the result that tumor response rate could be improved by reduction the interrupted days of radiation therapy through the schedule modification of Cisplatin. In future, if such clinical studies were performed prospectively, they would be significant studies to validate this analysis, and it is thought that it may bring out an important influence on determining the standard treatment in patients with locally advanced nasopharyngeal cancer.

Recently, it has been known that overexpression of epidermal growth factor receptor (EGFR) is associated with poor prognosis. And many clinical trials to evaluate the anticancer efficacy of the targeted agents against EGFR have demonstrated encouraging results, too $(22,23)$. Together with this, innovative treatment techniques such as intensity modulated radiation therapy (IMRT) are expected to bring more improved clinical outcomes in the near future (15).

\section{CONCLUSION}

In current study, treatment outcomes such as tumor response rate, progression-free survival, and overall survival were analyzed retrospectively in patients with locally advanced nasopharyngeal cancer. Schedule modification of Cisplatin from $100 \mathrm{mg} 3$ weeks to weekly regimen resulted in improved compliance and treatment outcome by the reduction of radiation interruption and treatment-related acute toxicities. 


\section{REFERENCES}

1. Wei WI, Sham JST. Nasopharyngeal carcinoma. Lancet. 2005; 365:2041-54.

2. Cho WC. Nasopharyngeal carcinoma: molecular biomarker discovery and progress. Mol Cancer. 2007;6:1.

3. Chan AT, Teo PM, Johnson PJ. Nasopharyngeal carcinoma. Ann Oncol. 2002;13:1007-15.

4. Qin DX, Hu YH, Yan JH, Xu G,Z Cai WM, Wu XL, et al. Analysis of 1,379 patients with nasopharyngeal carcinoma treated by radiation. Cancer. 1988;61:1117-24.

5. Al-Sarraf M, LeBlanc M, Giri PG, Fu KK, Cooper J, Vuong $\mathrm{T}$, et al. Chemoradiotherapy versus radiotherapy in patients with advanced nasopharyngeal cancer: Phase III randomized Intergroup study 0099. J Clin Oncol. 1998;16:1310-7.

6. Vokes EE, Weichselbaum RR. Concomitant chemoradiotherapy: Rationale and clinical experience in patients with solid tumors. J Clin Oncol. 1990;8:911-34.

7. Al-Sarraf M. Treatment of locally advanced head and neck cancer: Historical and critical review. Cancer control. 2002; 9:387-99.

8. Wee J, Tan EH, Tai BC, Wong HB, Leong SS, Tan T, et al. Randomized trial of radiotherapy versus concurrent chemoradiotherapy followed by adjuvant chemotherapy in patients with American Joint Committee on Cancer/International Union Against Cancer stage III and IV nasopharyngeal cancer of the endemic variety. J Clin Oncol. 2005;23:6730-8.

9. Lin JC, Jan JS, Hsu CY, Liang WM, Jiang RS, Wang WY. Phase III study of concurrent chemoradiotherapy versus radiotherapy alone for advanced nasopharyngeal carcinoma: positive effect on overall and progression-free survival. J Clin Oncol. 2003;21:631-7.

10. Baujat B, Audry H, Bourhis J, Chan AT. Chemotherapy as an adjunct to radiotherapy in locally advanced nasopharyngeal carcinoma (Review). 2007 The Cochrane Collaboration.

11. Kwong DL, Sham JS, Chua DT, Choy DT, Au GK, Wu PMl. The effect of interruptions and prolonged treatment time in radiotherapy for nasopharyngeal carcinoma. Int $\mathrm{J}$ Radiati Oncol Biol Phys. 1997;39:703-10.

12. Isobe $\mathrm{K}$, Kawakami $\mathrm{H}$, Uno $\mathrm{T}$, Yasuda $\mathrm{S}$, Aruga $\mathrm{T}$, Ueno $\mathrm{N}$, et al. Concurrent chemoradiotherapy for locally advanced nasopharyngeal carcinoma: is intergroup study 0099 feasible in
Japanese patients? Jpn J Clin Oncol. 2003;33:497-500.

13. Kwong DL, Sham JS, Au GK, Chua DT, Kwong PW, Cheng $\mathrm{AC}$, et al. Concurrent and adjuvant chemotherapy for nasopharyngeal carcinoma: A factorial study. J Clin Oncol. 2004; 22:2643-53.

14. Chitapanarux I, Lorvidhaya V, Kamnerdsupaphon P, Sumitsawan Y, Tharavichitkul E, Sukthomya V, et al. Chemoradiation comparing cisplatin versus carboplatin in locally advanced nasopharyngeal cancer: Randomized, non-inferiority, open trial. Eur J Cancer. 2007;43:1399-406.

15. O'Meara WP, Lee N. Advances in nasopharyngeal carcinoma. Curr Opin Oncol. 2005;17:225-30.

16. RTOG acute radiation morbidity scoring criteria < http://www. rtog.org/ members/toxicity/acute.html>.

17. Cheng SH, Jian JJ, Tsai SY, Yen KL, Chu NM, Chan NM, et al. Long-term survival of nasopharyngeal carcinoma following concomitant radiotherapy and chemotherapy. Int J Radiat Oncol Biol Phys. 2000;48:1323-30.

18. Bahl M, Siu LL, Pond GR, Kim J, Tannock IF, Bayley A, et al. Tolerability of the Intergroup 0099 (INT 0099) regimen in locally advanced nasopharyngeal cancer with a focus on patients' nutritional status. Int J Radiat Oncol Biol Phys. 2004;60:1127-36.

19. Chen YP, Tsang NM, Tseng CK, Lin SY. Causes of interruption of radiotherapy in nasopharyngeal carcinoma patients in Taiwan. Jpn J Clin Oncol. 2000;30:230-4.

20. Reddy SP, Raslan WF, Gooneratne S, Kathuria S Marks JE. Prognostic significance of keratinization in nasopharyngeal carcinoma. Am J Otolaryngol. 1995;16:103-8.

21. Marks JE, Phillips JL, Menck HR. The national cancer data base report on the relationship of race and national origin to the histology of nasopharyngeal carcinoma. Cancer. 1998;83: 582-8.

22. Chua DT, Nicholls JM, Sham JS, Au GK. Prognostic value of epidermal growth factor receptor expression in patients with advanced stage nasopharyngeal carcinoma treated with induction chemotherapy and radiotherapy. Int J Radiat Oncol Biol Phys. 2004;59:11-20.

23. Chan AT, Hsu MM, Goh BC, Hui EP, Liu TW, Millward MJ, et al. Multicenter, phase II study of cetuximab in combination with carboplatin in patient with recurrent or metastatic nasopharyngeal carcinoma. J Clin Oncol. 2005;23:3568-76.

24. Hak Choy. Chemoradiation in cancer therapy. 1st ed. New Jersey: Humana Press; 2003. p. 9-50. 